\title{
Pylephlebitis: An infrequently recognized complication of intra-abdominal infection in the absence of detectable pathogens
}

\author{
Ekie G. Vázquez, Antonio R. Soto, Doris H. Toro \\ Veteran's Administration Caribbean Healthcare System, San Juan, Puerto Rico
}

Received: December 9, 2015

Accepted: January 6, 2016

Online Published: January 21, 2016

DOI: $10.5430 /$ crim.v3n1p60

URL: http://dx.doi.org/10.5430/crim.v3n1p60

\begin{abstract}
Pylephlebitis, also known as infective suppurative thrombosis of the portal vein, is a rare complication of intra-abdominal infections. Although pylephlebitis has been increasingly recognized due to advancement in imaging studies, it still carries a high mortality rate despite early identification and adequate treatment. In this case report, we describe a case of a 60-year-old man who presented with a two-week-history of general malaise, anorexia, fever and right-sided abdominal pain. Imaging studies revealed acute portal vein thrombosis associated to multiple non-enhancing liver lesions compatible with liver abscesses. The infection that lead to this dreaded complication was not identified at the time of presentation. Although liver abscesses resolved after six weeks of appropriate antibiotic treatment, portal vein thrombosis remained.
\end{abstract}

Key Words: Pylephlebitis, Abdominal infection, Infective suppurative thrombosis of the portal vein

\section{INTRODUCTION}

Abdominal pain is a very common complaint for which patients seek medical attention. The evaluation of a patient with abdominal pain is not an easy task; many etiologies need to be considered depending on patient's clinical presentation. When accompanied by fever and chills, it should raise concerns for infectious etiologies requiring prompt evaluation. Timely evaluation and initiation of proper antibiotic treatment may prevent complications such as liver abscesses and infective suppurative thrombosis of the portal vein. The latter is a rare complication of intra-abdominal infections, which conveys a high mortality and long-term consequences.

\section{CASE}

We present a case of a 60 -year-old hispanic man who presented to the emergency department (ED) complaining of a 2-week-history of general malaise, anorexia, fever and chills associated to right sided abdominal pain. He denied similar episodes in the past, change in bowel habits, dyspepsia and food intolerance. He reported past medical history of diabetes mellitus type II, arterial hypertension, obesity, coronary artery disease, and previous ischemic cerebrovascular accident. Vital signs were essentially normal (temperature of $98.5 \mathrm{~F}$, blood pressure 130/70, heart rate of 75 with a respiratory rate of 12). His physical examination revealed a hypoactive, chronically ill looking man. The oral mucosa was dry. The skin and sclera were icteric. Abdominal examination revealed an enlarged liver $(16 \mathrm{~cm})$ with tenderness to superficial and deep palpation.

Analysis from both clinical chemistry and hematology panel showed (see Tables $1 \& 2$ ) leukocytosis (WBC $22.0 \times 10^{3}$

*Correspondence: Ekie Vázquez; Email: Ekie.Vazquez-Maldonado@va.gov; Address: 10 Casia Street San Juan, PR 00921-3201, Puerto Rico. 
$\mu \mathrm{l})$, increased alkaline phosphatase of $203 \mathrm{U} / \mathrm{L}$, hyperbilirubinemia of $5.5 \mathrm{mg} / \mathrm{dl}$, with direct predominance $(3.6 \mathrm{mg} / \mathrm{dl})$, and elevated liver enzymes $(\mathrm{AST}=81 \mathrm{U} / \mathrm{L}, \mathrm{ALT}=45 \mathrm{U} / \mathrm{L})$.

Table 1. Complete blood count

\begin{tabular}{llll}
\hline Hematology & $\mathbf{1 / 1 0 / 1 2}$ & Units & Ref. Range \\
\hline WBC & $22.6 \mathrm{H}$ & $\times 10^{-3}$ & $4.28-9.3$ \\
RBC & 3.8 & $\times 10^{-6}$ & $4.7-6.1$ \\
HGB & $11.3 \mathrm{~L}$ & $\mathrm{~g} / \mathrm{dl}$ & $12.6-17.8$ \\
HCT & $31.7 \mathrm{~L}$ & $\%$ & $37.9-54.5$ \\
MCV & $83.4 \mathrm{~L}$ & $\mathrm{FI}$ & $80.6-103.7$ \\
RDW & 12.5 & $\%$ & $11-14$ \\
PLT & 183 & $\times 10^{-3}$ & $155-371$ \\
BANDS & 8 & $\%$ & 0 \\
SEGS & 74 & $\%$ & $34-74$ \\
LYMPHS & 2 & $\%$ & $16.9-47.4$ \\
MONOS & 13 & $\%$ & $4-12$ \\
\hline
\end{tabular}

Table 2. Chemistry panel

\begin{tabular}{llll}
\hline CHEM & $\mathbf{1 / 1 0 / 1 2}$ & UNITS & REF.RANGE \\
\hline GLUCOSE & $63 \mathrm{~L}$ & $\mathrm{mg} / \mathrm{dl}$ & $72-128$ \\
$\mathrm{BUN}$ & 21.7 & $\mathrm{mg} / \mathrm{dl}$ & $10-26$ \\
$\mathrm{CREA}$ & 0.95 & $\mathrm{mg} / \mathrm{dl}$ & $0.7-1.5$ \\
$\mathrm{Na}^{+}$ & $130 \mathrm{~L}$ & $\mathrm{eEq} / \mathrm{L}$ & $135-145$ \\
$\mathrm{~K}^{+}$ & 4.8 & $\mathrm{eEq} / \mathrm{L}$ & $3.5-5$ \\
$\mathrm{Cl}^{-}$ & $98 \mathrm{~L}$ & $\mathrm{eEq} / \mathrm{L}$ & $100-110$ \\
$\mathrm{CO}_{2}$ & 23 & $\mathrm{eEq} / \mathrm{L}$ & $24-32$ \\
$\mathrm{SGPT}$ & $45 \mathrm{H}$ & $\mathrm{U} / \mathrm{L}$ & $0-45$ \\
$\mathrm{SGOT}$ & $81 \mathrm{H}$ & $\mathrm{U} / \mathrm{L}$ & $0-40$ \\
$\mathrm{ALP}$ & $203 \mathrm{H}$ & $\mathrm{U} / \mathrm{L}$ & $30-115$ \\
$\mathrm{~T} . \mathrm{BILI}$ & $5.48 \mathrm{H}$ & $\mathrm{mg} / \mathrm{dl}$ & $0.2-1.3$ \\
$\mathrm{D} . \mathrm{BILI}$ & $3.63 \mathrm{H}$ & $\mathrm{mg} / \mathrm{dl}$ & $0-0.2$ \\
$\mathrm{EGFR}$ & 81 & $\mathrm{ml} / \mathrm{min}$ & $\geq 60$ \\
$\mathrm{Ca}$ & 8.5 & $\mathrm{mg} / \mathrm{dl}$ & $8.5-10.5$ \\
$\mathrm{ALB}$ & $2.4 \mathrm{~L}$ & $\mathrm{~g} / \mathrm{dl}$ & $2.6-5.2$ \\
$\mathrm{ING}$ PHO & $0.8 \mathrm{~L}$ & $\mathrm{mg} / \mathrm{dl}$ & $2.5-4.5$ \\
$\mathrm{Mg}$ & 2.3 & $\mathrm{mg} / \mathrm{dl}$ & $1.8-2.4$ \\
$\mathrm{CPK}$ & 59 & $\mathrm{U} / \mathrm{L}$ & $5-169$ \\
\hline
\end{tabular}

Non-contrast abdominal computerized tomography (CT) demonstrated multiple low attenuation lesions throughout the liver parenchyma. The patient was started on empiric broad-spectrum intravenous (IV) antibiotic therapy with piperacillin-tazobactam and admitted to internal medicine ward for further management. Gastroenterology consultant recommended a triphasic abdominal CT with liver protocol (see Figure 1), which was remarkable for a left liver lobe enhancing mass, associated to complete right portal vein thrombosis and a wedge shaped perfusion defect on the venous phase.

Published by Sciedu Press

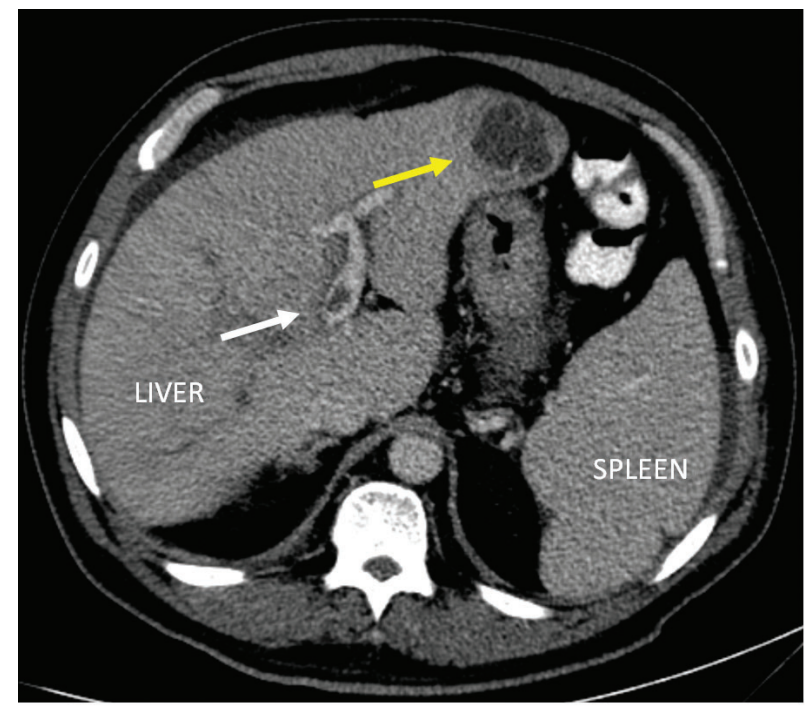

Figure 1. Triphasic abdominal CT with liver protocol revealed hepatomegaly with markedly heterogeneous parenchyma and enhancing mass at the left liver lobe (yellow arrow) with associated portal thrombosis (white arrow) and equivocal additional satellite lesions at the right liver lobe, prior to antibiotic therapy. Splenomegaly also was shown with findings suggestive of portal hypertension and ascites

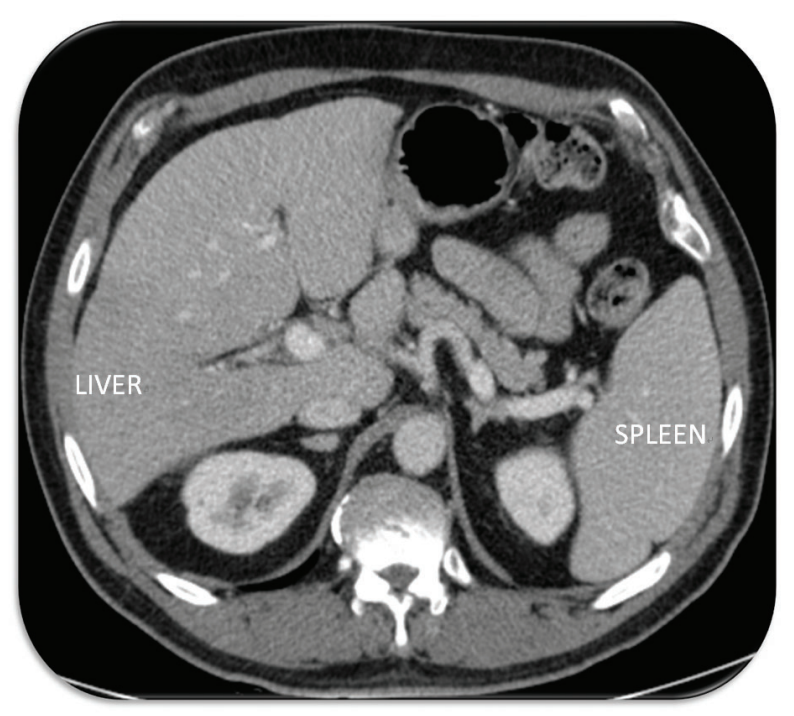

Figure 2. CT abdominal with IV contrast was done six week after completion of therapy for evaluation of interval changes. CT showed hepatomegaly, cirrhosis, with interval resolution of a low attenuation lesion at the lateral segment of the left hepatic lobe. Chronic portal vein thrombosis and splenomegaly

There were no bowel wall abnormalities. The gallbladder was described as normal, without stones. CT-guided lesion aspirate obtained purulent material consistent with liver abscess. Cytological analysis including HIV test, hepatitis 
profile, gammaglobulin levels, eosinophils, ova and parasites, Schistosoma, Strongyloides, E. Histolytica and acid fast bacilli were negative. Cultures from the aspirate as well were negative including areboic, anaerobic and fungi cultures. Blood cultures were also negative as well. The patient completed 6 weeks of antibiotic therapy with subsequent normalization of liver enzymes, alkaline phosphatase and bilirubin. Despite a thorough evaluation, which included a colonoscopy, the source of liver abscess and pylephlebitis was not identified. Follow up images after 6 weeks of antibiotic therapy (see Figure 2) showed resolution of liver abscesses, nevertheless portal vein thrombosis remained. Standard laboratory analysis after completions of therapy were remarkable for normal white blood cell counts, normalization of alkaline phosphatase levels as well as bilirubin and liver enzymes.

\section{Discussion}

Pylephlebitis is an infectious process that begins at small veins which can extend if left untreated into larger veins leading to septic thrombosis of the portal vein. ${ }^{[1]}$ There has been an increased recognition of this complication with the greater use and advances in diagnostic imaging; but despite better recognition, the presence of pylephlebitis still carries a high morbidity and mortality despite adequate treatment. ${ }^{[1-4]}$ Symptoms of infective suppurative thrombosis of the portal vein are non-specific. ${ }^{[1]}$ This rare condition usually arises from an intra-abdominal infectious process such as divertic- ulitis, abscess, colitis, appendicitis or cholangitis; however, the source of infection is not identified in up to $70 \%$ of the cases, such as in our patient. ${ }^{[1,3,4]}$ Blood cultures are frequently positive and usual isolated pathogens include $B$. fragilis, E. coli and P. mirabilis, though polymicrobial involvement is still very common. ${ }^{[1]}$ Prompt recognition and treatment of this condition is crucial since serious complications may arise such as bowel ischemia, pyogenic liver abscess, portal hypertension and/or death. ${ }^{[2,4]}$ Upon clinical suspicion, broad spectrum empiric antibiotic therapy should be started and continued for at least 4-6 weeks if diagnosis is confirmed. ${ }^{[1,2]}$

In summary, pylephlebitis is an uncommon complication of an intrabdominal infectious process, which can be fatal if not diagnosed and treated promptly. Diverticulitis, appendicitis and hepatic abscess are the most common conditions associated to pylephlebitis, however other etiologies such a malignancies need to be ruled out. Antibiotics against gram negative and anaerobic bacteria have been the gold standard for treating pylephlebitis. In recent years mortality has decreased significantly (up to $25 \%$ ), due to early recognition, imaging studies and antibiotic therapy. ${ }^{[5]}$ Current data is inconclusive whether anticoagulation should instituted as standard management for pylephlebitis, nonetheless clinical trials have shown improvement of patient's outcome in those who experience mesenteric thrombosis, ischemia or an underlying hypercoagulable state. ${ }^{[1,6]}$

\section{REFERENCES}

[1] Wong K, Weisman DS, Patrice KN. Pylephlebitis: A rare complication of an intra-abdominal infection. Journal of Community Hospital Internal Medicine Perspectives. 2013; 3(2): 1-4. PMid:23882407 http://dx.doi.org/10.3402/jchimp.v3i2.20732

[2] Plemmons RM, Dooley DP, Longfield RN. Septic Thrombophlebitis of the Portal Vein (Pylephlebitis): Diagnosis and Management in the Modern Era. Clinical Infectious Disease. 1995; 21(5): 1114-1120. http://dx.doi.org/10.1093/clinids/21.5.1114

[3] Van De Wauwer C, Irvin TT. Pylephlebitis due to Perforated Diverticulitis. Acta Chirurgia Belgica. 2005; 105(2): 229-230. PMid:15906925
[4] Gajendran M, Muniraj T, Yassin M, et al. Diverticulitis complicated by pylephlebitis: a case report. Journal of Medical Case Reports. 2011; 5: 514. PMid:21985694 http://dx.doi.org/10.1186/1 752-1947-5-514

[5] Baril N, Wren S, Radin R, et al. The role of anticoagulation in pylephlebitis. The American Journal of Surgery. 1996; 172(5): 449-4453. http://dx.doi.org/10.1016/S0002-9610(96)00220-6

[6] Sanyal AJ, Chopra S, Travis AC. Acute portal vein thrombosis in adults: Clinical manifestation, diagnosis and management. Up To Date. 2013. Available from: http://www. uptodate.com/conte nts/acute-portal-vein-thrombosis-in-adults-clinica 1-manifestations-diagnosis-and-management 